provided a narrow gap between the cathode and the ceramic to avoid a probable one point contact between cathode and ceramic in the absence of a perfect fit. Because all of the kovar cathode tests had been run with sealed-on glass insulation, a control test was made in which the relative deposit rates of glass- and porcelain-insulated kovar cathodes were measured (table 1, group V, tubes No. 29, 31, 33, 35). This control test showed a somewhat larger arc rate in the latter case, which at first thought appears contrary to the practice, generally accepted in tube development, of discouraging arc back by just such separation of a negative electrode from its insulation. The practice arose after the study by Issendorf, Schenkel, and Seeliger ${ }^{4}$ of the effect on arc back of various insulating materials in contact with a negative electrode. In the present case, the explanation of the apparent discrepancy probably lies in the difference of insulator material, and use of quartz, alundum, or even glass instead of porcelain might have avoided the discrepancy. The question was not pursued, since the improvement derived from use of 18-8 stainless steel cathodes far outweighed the disadvantage of the insulator choice, leaving no question as to the importance of the choice of cathode material.

The remedial steps suggested by this work are clear. Distributed capacity should be reduced as far as possible. Pulsing the discharge should be avoided when permissible, and the cathode current density should be as low as possible. Ideally, water vapor or other active gas or vapor should be omitted from the tube. This is an unobtainable ideal in most cases; an electronegative component is usually required in a TR tube in order to keep recovery time adequately short. The steps mentioned so far are generally limited in their feasibility by the particular application of the tube. One factor, the cathode material is usually not so determined. It has been shown that selection of this material is a critical factor in determining the life of a tube. It should therefore be possible to extend the life of TR tubes operating under the assumed conditions, where life is prematurely ended by keep-alive short circuit, by substitution of a ceramically insulated 18-8 stainless steel cathode for the customary glass insulated kovar cathode. The results described here might well be of value in the design of any electron tubes wherein high current density glow discharges are maintained between electrodes in close proximity.

The generous and valuable advice of John E. White, Chief of the Bureau's Electron Tube Laboratory, and of Wm. G. Dow of the University of Michigan is gratefully acknowledged.

Washington, May 11, 1950.

\title{
Test Mixtures for Distillation at Atmospheric and Reduced Pressure'
}

\author{
By Charles B. Willingham ${ }^{2}$ and Vincent A. Sedlak ${ }^{3}$
}

\begin{abstract}
In this paper are reported values for the separation factor for distillation for each of the three pairs of diethylbenzenes for pressures from 30 to $800 \mathrm{~mm} \mathrm{Hg}$. Also given are data on the refractive index as a function of composition for the system 1,2-diethylbenzene and 1,3-diethylbenzene.
\end{abstract}

A mixture of 1,2-diethylbenzene and 1,3-diethylbenzene was selected on the basis of boiling point, separation factor, and expected ideality for use as a test mixture for investigating the separating efficiency, at pressures in the range from 30 to $760 \mathrm{~mm}$ $\mathrm{Hg}$, of the rotary concentric-tube $[1,2]^{4}$ and other distilling columns [3]. As there is a difference of 0.0078 in the values of the refractive index, $n_{D}$ at $25^{\circ} \mathrm{C}$, of the two pure components, it is possible to analyze this mixture refractometrically within one and one-half percent of the composition from measurements of refractive index made to the nearest 0.0001 .

1 This investigation was performed at the National Bureau of Standards as part of the American Petroleum Institute Research Project 6 on the Analysis, Purification, and Properties of Hydrocarbons.

2 Now with the Mellon Institute, Pittsburgh, Pa.; formerly with the National Bureau of Standards.

${ }_{3}$ Now with the American Petroleum Institute Research Project 6 at the Carnegie Institute of Technology; formerly a Research Associate at the National Bureau of Standards.

4 Figures in brackets indicate the literature references at the end of this paper.
Accordingly, known mixtures of 1,2-diethylbenzene and 1,3-diethylbenzene, having compositions near 25,50 , and 75 percent of the former, were made up accurately by weight, by using samples of purified compounds corresponding to the material prepared for standard samples [4]. Measurements of the differences in the refractive index of these mixtures and of pure 1,2-diethylbenzene, with respect to pure 1,3-diethylbenzene, were made by using a modified Abbe-type precision refractometer [5]. The results, expressed as the difference between the refractive index of the mixture and of 1,3-diethylbenzene, are as follows:

For 1,3-diethylbenzene and 1,2-diethylbenzene, at $25^{\circ} \mathrm{C}$

$$
\begin{gathered}
n_{D}(\text { mixture })-n_{D}(1,3 \text {-diethylbenzene })= \\
0.00782 N-0.00008 N^{2},
\end{gathered}
$$


where $n_{D}$ is the refractive index, and $N$ is the mole fraction of 1,2-diethylbenzene in the mixture. The foregoing equation reproduces the experimentally observed values of refractive index within \pm 0.0001 .

The number of equivalent theoretical plates at total reflux in a rectifying section may be calculated from the equation $[6,7]$

$$
n+1=[1 / \log \alpha] \log \left[\left(N_{A} / N_{B}\right)^{\text {head }} /\left(N_{A} / N_{B}\right)^{\text {pot }}\right],
$$

where

$$
\alpha=P_{A}^{*} / P_{B}^{*} .
$$

$P^{*}$ is the vapor pressure of the given component in the pure state at the mean temperature, $N$ is the mole fraction of the given component in the liquid phase in the head or pot as indicated, and $A$ and $B$ represent the more volatile and the less volatile component, respectively, in an ideal binary solution.

Binary mixtures of the isomeric diethylbenzenes are expected to be substantially ideal as regards the relation of vapor pressure to composition (high efficiency distillations of aromatic concentrates have disclosed no nonideality between close-boiling isomeric aromatic hydrocarbons) $[8,9]$. The data on the vapor pressure as a function of temperature of the pure components recently reported from this laboratory [10] were reduced for each of the three pairs of isomers to give values of $\alpha$ at mean temperatures corresponding to average vapor pressures between 30 and $800 \mathrm{~mm} \mathrm{Hg}$. For convenience in evaluating the separating power of distilling columns, the separation factor, $1 / \log _{10} \alpha$, as a function of the logarithm of the average pressure for the components, between 30 and $800 \mathrm{~mm} \mathrm{Hg}$ is presented graphically in figures 1 and 2 .

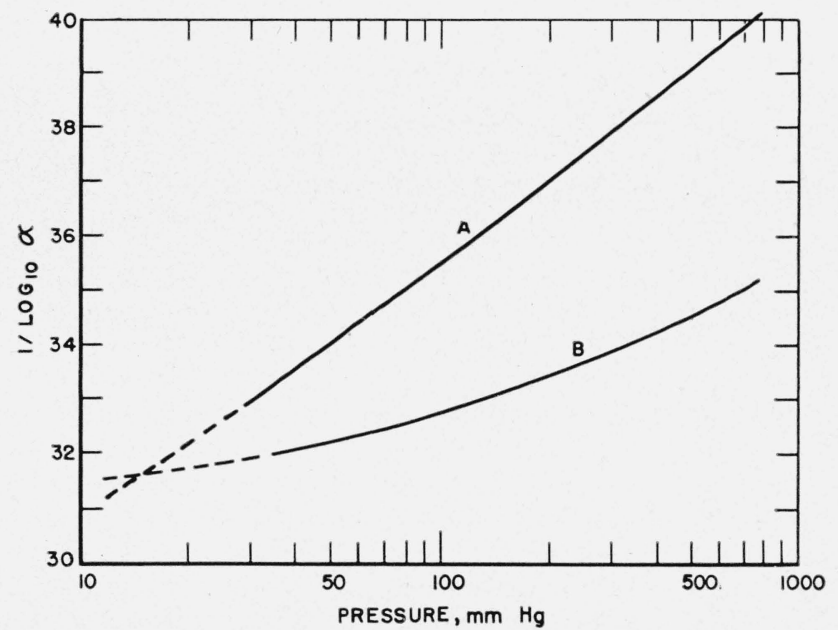

FIGURE 1. Values of the separation factor as a function of the logarithm of the average pressure. The scale of ordinates gives the values of the separation factor, $1 / \log _{10} \alpha$, and the
scale of abscissas gives the values of the logarithm of the average pressure in $\mathrm{mm}$ $\mathrm{scale}$ of abscissas gives the values of the logarithm of the average pressure in $\mathrm{mm}$
$\mathrm{Hg}$. Curve $A$ is for the mixture of 1,3-diethylbenzene and 1,2-diethylbenzene. Curve $B$ is for the mixture of 1,3-diethylbenzene and 1,4-diethylbenzene.

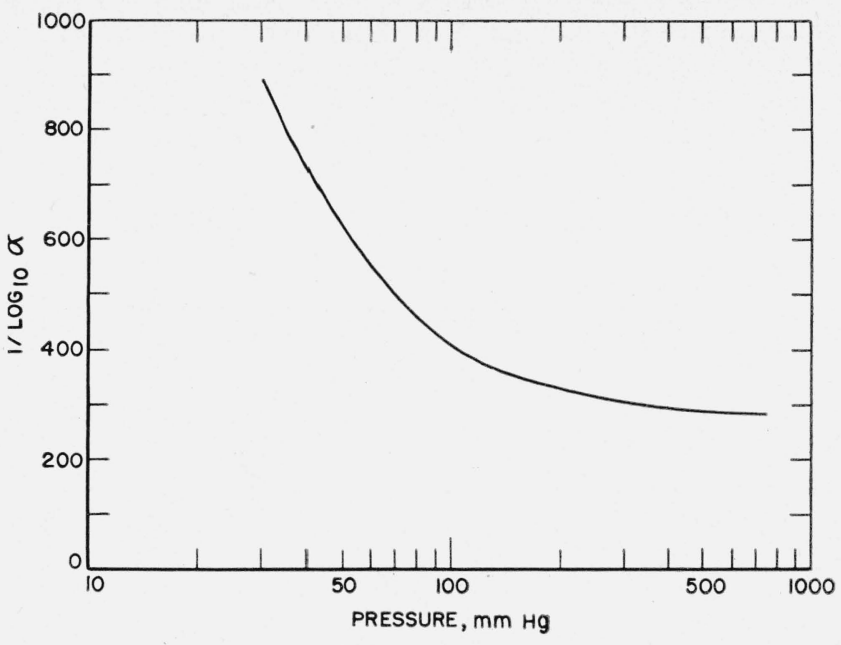

FIGURE 2. Values of the separation factor as a function of the logarithm of the average pressure.

The scale of ordinates gives the values of the separation factor, $1 / \log _{10} \alpha$, and the scale of abscissas gives the values of the logarithm of the average pressure in $\mathrm{mm}$ $\mathrm{Hg}$ for the mixture of 1,2-diethylbenzene and 1,4-diethylbenzene.

At $1 \mathrm{~atm}$ pressure, the values of the boiling points for the three isomers are as follows [10]:

$$
\begin{aligned}
& \text { 1,4-diethylbenzene, } 183.75^{\circ} \mathrm{C} \text {; } \\
& \text { 1,2-diethylbenzene, } 183.42^{\circ} \mathrm{C} \text {; } \\
& \text { 1,3-diethylbenzene, } 181.10^{\circ} \mathrm{C} \text {. }
\end{aligned}
$$

The values of $\alpha$ at the mean boiling point at $1 \mathrm{~atm}$ for each of the three pairs of isomers are calculated from the data of reference [10] as follows:

\section{1,3-diethylbenzene and 1,4-diethylbenzene, 1.0677;}

1,2-diethylbenzene and 1,3-diethylbenzene, 1.0591;

\section{1,2-diethylbenzene and 1,4-diethylbenzene, 1.0081.}

Although only one of the three pairs of isomers may be analyzed as a mixture refractometrically, all three pairs may be analyzed by infrared spectrcphotometry [11], since purified standard samples of each of the three diethylbenzenes are now arailable [4] for spectrometer calibration.

Grateful acknowledgment is made to Frederick D. Rossini for his suggestions in the course of this investigation, and to Alphonse F. Forziati for the measurements of refractive index.

[1] C. B. Willingham, V. A. Sedlak, J. W. Westhaver, and F. D. Rossini, Ind. Eng. Chem. 39, 706 (1947).

[2] C. B. Willingham, V. A. Sedlak, J. W. Westhaver, and F. D. Rossini, Test of the rotary concentric-tube distilling column at reduced pressure. 
[3] C. B. Willingham and F. D. Rossini, J. Research NBS 37, 15 (1946) RP1724.

[4] A. J. Streiff, J. C. Zimmerman, L. F. Soule, M. T. Butt, V. A. Sedlak, C. B. Willingham, and F. D. Rossini, J. Research NBS 41, 323 (1948) RP1929.

[5] A. F. Forziati, J. Research NBS 44, 373 (1950) RP2085.

[6] M. R. Fenske, Ind. Eng. Chem. 24, 482 (1932).

[7] M. R. Fenske, Science of Petroleum, II, 1629. (Oxford Univ. Press, London, 1938).

[8] A. F. Forziati, C. B. Willingham, B. J. Mair, and F. D. Rossini, J. Research NBS 32, 11 (1944) RP1571.

[9] A. F. Forziati and F. D. Rossini, J. Research NBS 39, 425 (1947) RP1839.
[10] A. F. Forziati, W. R. Norris, and F. D. Rossini, J. Research NBS 43, 555 (1949) RP2049.

[11] American Petroleum Institute Research Project 44. National Bureau of Standards. Catalog of Infrared Spectral Data, Serial No. 326, 1,2-diethylbenzene, contributed by the Socony Vacuum Oil Co., and Serial Nos. 331, 332, 333 for the three isomers of diethylbenzene, contributed by the Anglo-Iranian Oil Co.

Washington, June 1, 1950.

\title{
Aging of Karakul and Seal Fur Skins
}

\author{
By Edward T. Steiner and Elizabeth R. Hosterman
}

\begin{abstract}
Physical and chemical data are presented for two types of fur skins of widely different dressings and characteristics to show that deterioration takes place when furs are stored under conditions considered noninjurious. There occur during storage an appreciable loss of strength amounting to as much as 25 to 40 percent, and a change in certain chemical properties. Pelts weakened by aging showed increased soluble nitrogen and water extractives and lower grease contents (petroleum ether extractives) than less aged skins. A fair correlation exists between the observed changes in chemical and physical properties. The presence and quantity of copper in the weakened pelts offer the only reasonable explanation for the cause and the extent of the deterioration.
\end{abstract}

\section{Introduction}

The value of dressed fur skins used annually in this country is estimated at several hundred million dollars. The more than moderate investment that is required in the purchase of fur garments does not always seem to be commensurate with the expected 2- to 10-year service life of most furs. By contrast, properly tanned leather may be expected to last from 10 to 40 years when not subjected to severe abrasion or chemical action. If the causes for the relatively short life span of the processed furs could be discovered and eliminated, there would result considerable economic advantage to the consumer, the distributor, and the manufacturer.

Various reasons have been offered for the relatively poor wearing qualities of fur garments. In the first place, the skins from most fur animals are naturally weak. Secondly, the procedures for dressing the skins are not so highly developed as those for making leather, primarily because of the purposes for which the furs are intended. In straight leather making, removal of the hair is required. The process of removing it tends to give better quality leather. With furs, the hair, of course, must be left on. Certain chemicals and tanning agents suitable for leather manufacture adversely affect fur quality and appearance, and cannot be used for fur dressing. It is the aim of fur dressers to keep the weight of the dressed pelts light, while the leather manufacturer usually strives to give weight to his product.

Carelessness on the part of the consumer is often responsible for the poor service given by furs. Improper drying after exposure to the elements, faulty cleaning methods, and storage in polluted atmospheres materially shorten the life of fabricated furs.
Such factors, however, do not account for the loss of strength of furs that have never been exposed to such conditions. Fur skins frequently become weak with age, apparently without cause.

The source of the deterioration is not apparent because the leather is concealed from view between the hair of the fur and the lining of the garment. The present work was undertaken to investigate the possible causes and extent of deterioration occurring in fur skins stored under conditions that would not be expected to cause losses of strength.

The Government's interest in furs centers about a protective and advisory role. It wishes to assist and cooperate in helping to bring about maintenance of a constant supply of fur animals, whether they be wild or captive. Through its agency, the Fish and Wildlife Service, Department of Interior, the Government compiles results of surveys of state agencies on the stock of various species and enacts legislation based on these statistics, regarding the "take" of the animals, in order to provide a balance between supply and preservation. Through another of its agencies, the Fur Farming Investigations, of the Department of Agriculture, economical methods of feeding, breeding, and management of fur animals in captivity are studied.

Examples of the work the Government has been doing along these lines include supervision of the annual catch of Alaskan fur seals off the Pribilof Islands and experimentation in the breeding of Karakul sheep.

Alaskan seal furs are furs of distinction and importance in the industry. By far the world's largest supply comes from the Pribilof Islands off the coast of Alaska. Until 1910 pelagic sealing, which means the killing of seals in water, almost resulted in the 